\title{
624 MORTALITY REDUCTION ASSOCIATED WITH PROACTIVE USE OF EMR-BASED ACUITY SCORE BY AN RN TEAM AT AN URBAN HOSPITAL
}

Michael Rothman, ${ }^{1}$ Joan Rimar, ${ }^{2}$ Sheila Coonan, ${ }^{3}$ Stephen Allegretto, ${ }^{2}$ Thomas Balcezak ${ }^{3}$. 'PeraHealth, United States; ${ }^{2}$ Yale New Haven Health System, United States; ${ }^{3}$ Yale-New Haven Hospital, United States

10.1136/bmjqs-2015-IHlabstracts.21

Background Early identification of clinical deterioration is critical in providing high quality care to hospitalized patients. Having invested heavily in electronic medical records, hospitals are leveraging EMR data to address failure to rescue occurrences. The Rothman Index (RI), an acuity score which provides a graphical view of patient condition using EMR vital signs, lab results and nursing assessments, can be used to monitor patient condition.

Objectives To determine if risk-adjusted mortality could be decreased with implementation of a nursing SWAT team, using RI graphs, to identify clinically deteriorating patients at a 350-bed hospital campus.

Methods Each shift the SWAT nurse reviewed the RI graphs of all adult medical-surgical patients and pro-actively rounded on patients with the lowest or sharply declining scores who were not in an ICU. The study had a 6-month control period and a 9 -month intervention period. A second control was provided by a sister campus for the 15 month period.

Results Mortality fell from $1.9 \%$ to $1.3 \%(p<.001)$ in a patient population of 24,637. The percent of highest risk

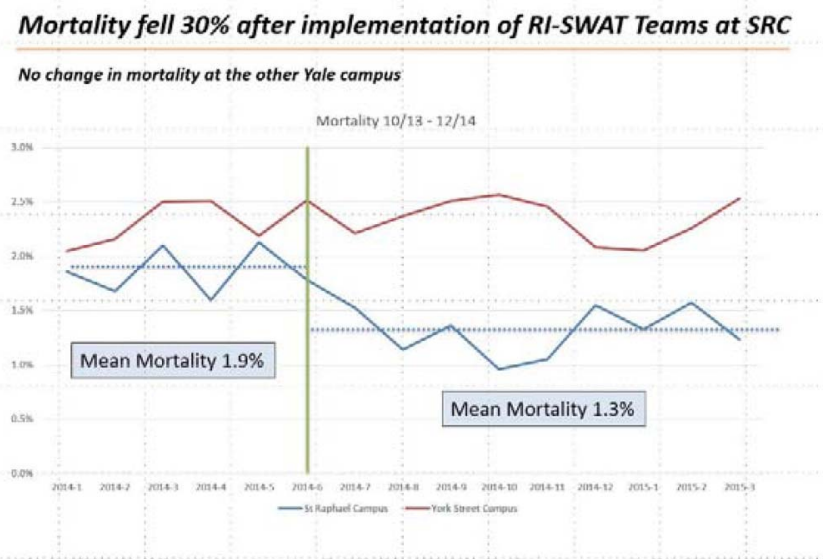

Figure 1 Mortality at two Yale campuses - 15 months. Mortality fell from $1.9 \%$ to $1.3 \%$ from the control to the intervention period (blue line). The mortality at the sister hospital was unchanged at $2.3 \%$ (red line). 
Risk rose and hospice discharges increased during the intervention

Discharges to hospice fell from $1.9 \%$ to $1.5 \%$

Percent High Risk Population (ROM 3 and 4) at SRC

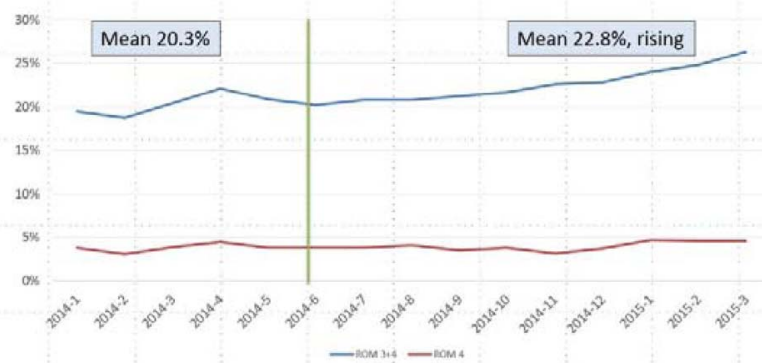

Figure 2 Risk of Mortality at SRC (intervention campus) - 15

months. While mortality fell, risk of mortality (Admit APR Risk Model) increased from the base period to the intervention period. In this model risk increases from ROM 1 to ROM 4. Typically, ROM 1 has a mortality rate of $<0.1 \%$, ROM 2 about $1 \%$, ROM 3 about $5 \%$. ROM 4 about $25 \%$. ROM 3 and ROM 4 rose from $20.3 \%$ of hospital patient population to $22.8 \%$. ROM 3 plus ROM 4 is shown as a blue line. ROM 4 alone is shown as a red line. Discharges to hospice also fell from $1.9 \%$ to $1.5 \%$.

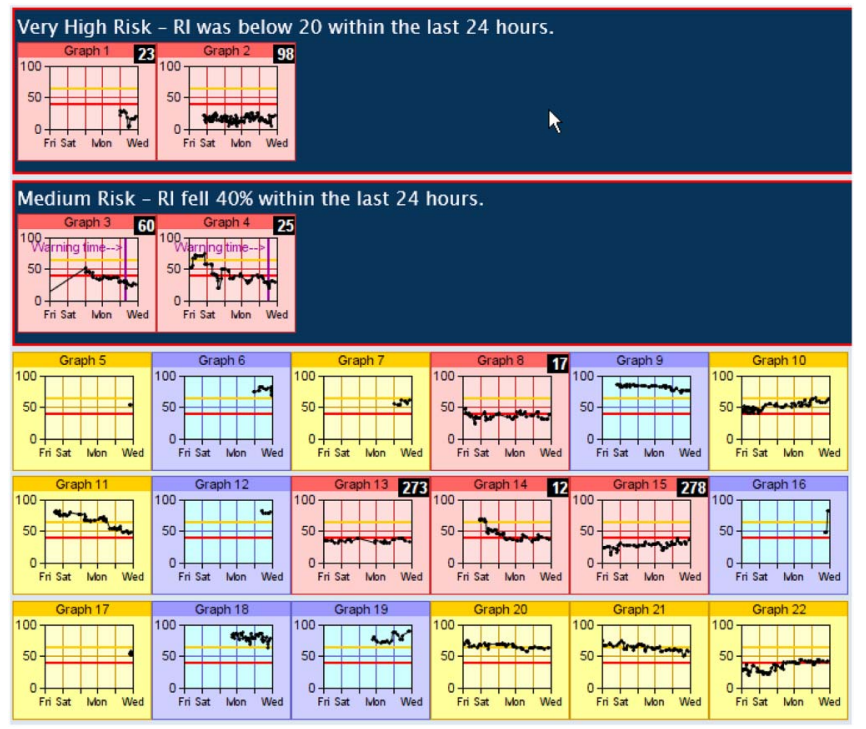

Figure 3 A typical array of RI Graphs which a SWAT nurse would view. Each box is a 5-day view of a patient's acuity. The graphs are color-coded by the last RI, with red being the highest acuity. An RI of 100 is for patients who are unimpaired. Lower numbers indicate greater risk. The top rows are for patient graphs where RI had either fallen below a critical number, or had fallen a certain percent in a given time period.

Table 1 Mortality rates and patient volume in the base period and in the intervention period at the two campuses York St Campus is the sister hospital. St Raphael Campus is the intervention hospital

\begin{tabular}{lllll}
\hline & Before 6 months & $\mathbf{N}$ & After 9 months & $\mathbf{N}$ \\
\hline York St Campus & $2.32 \%$ & 21873 & $2.34 \%$ & 33118 \\
St Raphael Campus & $1.85 \%$ & 9886 & $1.30 \%$ & 14751 \\
\hline
\end{tabular}

patients (admit APR Risk of Mortality 3 and 4) increased (20.3\% to $22.8 \%)$ and the percent of discharges to hospice decreased $(1.9 \%$ to $1.5 \%)$ during the intervention period. The mortality at the sister campus remained steady at $2.3 \%$ (population 55,991) during the 15 -months.

Conclusions Use of an EMR-based acuity index by a new, dedicated SWAT team was associated with a $30 \%$ mortality reduction. This represents an important use of the EMR to improve deterioration identification and thus quality of care. 